\title{
The potential of androgenic alopecia management from plant derivatives
}

\author{
1," Noor, N.M., ${ }^{2}$ Nazri, N.Z., ${ }^{2}$ Mohamad-Salam, N.A., ${ }^{1}$ Abdul-Rasid, Z.I., ${ }^{1,3}$ Hasham, R. and \\ ${ }^{1,3}$ Abdul-Aziz, A. \\ ${ }^{1}$ Cosmeceutical and Fragrance Laboratory, Institute of Bioproduct Development (N22), Universiti \\ Teknologi Malaysia, 81310 UTM Johor Bahru, Johor, Malaysia \\ ${ }^{2}$ Faculty of Pharmacy, Universiti Teknologi MARA (UiTM), 42300 Bandar Puncak Alam, Selangor, \\ Malaysia \\ ${ }^{3}$ School of Chemical and Energy Engineering, Faculty of Engineering, Universiti Teknologi Malaysia, \\ 81310 UTM Johor Bahru, Johor, Malaysia
}

\author{
Article history: \\ Received: 5 March 2020 \\ Received in revised form: 27 \\ May 2020 \\ Accepted: 30 July 2020 \\ Available Online: 9 August \\ 2020
}

\section{Keywords:}

Hair loss,

Androgenic alopecia,

Dihydrotestosterone,

$5 \alpha$-reductase,

Plant derivatives

\section{DOI:}

https://doi.org/10.26656/fr.2017.4(S2).S09

\begin{abstract}
Hair loss or alopecia is a common dermatological issue that can affect millions of human population of all ages and both gender, male and female. Frequently, alopecia has been found to be associated with significant adverse effects or reduction of psychological and self-esteem. Consequently, this may lead to psychological problems such as depression and anxiety, thus it may negatively impact the quality of life as well. There are several types of hair loss including androgenetic alopecia (AGA), alopecia areata (AA), alopecia totalis (AT), Alopecia Universalis (AU), cicatricial alopecia (CA), senescent alopecia (SA), traction alopecia (TA) and telogen effluvium. However, this review will focus on the androgenic alopecia only. Androgenic alopecia (AGA) also known as male pattern baldness is referred to as hair loss that often occurs in men after puberty caused by the androgen. In addition, this review will discuss on the hair growth cycles and their mechanism on the androgenic alopecia and lastly the management of androgenic alopecia using plant derivatives and methods used in order to prolong the efficacy of androgenetic alopecia treatment.
\end{abstract}

\section{Introduction}

Generally, the human may have between 90,000 and 150,000 of hairs on their head and loss between 50 and 100 per day (Erdoğan, 2017; Sengar et al., 2018). Though, the hair loss does not noticeable, as the new hair will grow at the same times. However, if the hair growth cycle was interrupted or the hair loss is more than 100 per day, then hair loss disorder that associated with pathological issues is seen (Shaikh et al., 2016). Hence, it is important to understand the causes of hair loss thoroughly as it can be the key part of the treatment or prevention of the alopecia. There are numbers of causal factors for alopecia to take place including the hereditary thinning, diseases, hormonal imbalance and stress, medication and nutrition. However, in this review, the focus will be on the androgenic alopecia. This review will discuss the anatomy and physiology of hair and it's growth cycle, causes of androgenic alopecia, and treatment and management of hair loss using plant derivatives. This review also will discuss on the application of nanoparticles as one of the delivery systems potentially used for androgenic alopecia treatment.

\section{Hair anatomy and physiology}

Hair mostly can be found on all mammals and not on other animals. Hair is a derivative of the epidermis which physically is thin, flexible tubes of a dead, fully keratinized cell. Hair from the macrostructural point of view, hair has varied in term the hair length, diameter, colour and cross-sectional shape (Buffoli et al., 2014). Hair serves as many functions in which the main function is to protect the surface of the skin and act as the thermoregulator. Human hair also helps in sweating thermoregulation and also act as a sensory organ. Besides, provide protection against UV radiation and elements, the hair also plays an important role as a psychological function for identity and self-confident in human. 
Hair consists of two different structure fibre which is follicle and hair shaft. The hair follicle is said as the living part of the hair that located under the skin while the hair shaft is fully keratinized dead part that can be found above or on the skin surface (Buffoli et al., 2014; Cooper, 2015; Erdoğan, 2017; Murphrey et al., 2020). The hair shaft is the hair that visible on the skin surface. Hair shaft consisted of three layers of keratin (a hardening protein) that are cuticle, cortex, and medulla. The cuticle is the outermost layer that is formed by tightly packed scales resemble roof shingles in an overlapping structure. Cuticle cell covers the hair from the root to the tip of the epidermis that also has important protective properties also act as barrier function against physical and chemical insults (Buffoli et al., 2014; Cooper, 2015). Next, the middle layer called cortex which makes up the majority of the hair shaft and the innermost layer is medulla. Both cortex and medulla contain pigmenting cell that responsible for the hair colour.

The essential part of the hair growth structure is follicle. The hair follicle is like a stocking-like structure that contains cells and connective tissue. The base of the follicle where the papilla can be found. The papilla contains tiny blood capillaries that help to give nutrition to the cells. The follicle basically consists of two distinct parts, the upper and lower part (Buffoli et al., 2014; Cooper, 2015). First, the upper part has infundibulum and isthmus. The infundibulum is the uppermost portion of the hair follicle from the opening of the sebaceous gland to the surface of the skin and the isthmus is the lower portion of the upper part that located between the openings of the sebaceous gland and the erector pili muscles. The lower part of the follicles consists of hair bulb and suprabulbar region where it has continuous cycles of regeneration (Erdoğan, 2017). The suprabulbar region of the follicle composes of three layers, outer root sheath, inner root sheath, and hair shaft. The outer root sheath act as the reservoir of stem cells, keratinocyte and melanocytes stem cells. The inner root sheath has three layers, Henle's layer, Huxley's layer, and cuticle layer. The inner root sheath cells produce keratins that give strength to support the growing hair shaft and guide the upward movement. The inner root sheath also acts as the separator that separates the hair shaft from the outers root sheath. The hair bulb is the stocking like structure that surrounds the papilla and germinal matrix that take nutrient from the capillaries around it. The follicular papilla is an essential source of growth factor that responsible for the hair growth cycle which the volume and secretory activity of follicular papilla with the number of matrix stem cells help determine the size of the anagen hair bulb, the duration of the anagen phase and the diameter of the hair shaft (Buffoli et al., 2014;
Cooper, 2015).

The whole of human body surface nearly covers with hairs except palms, soles and mucosal region (lips and external genitalia). There are three basic hair types on the human body which are vellus, intermediate and terminal hair (Buffoli et al., 2014; Cooper, 2015). Vellus hair covering the majority part of body surface both children and adults. Vellus hair are characterized with short $(<2$ $\mathrm{mm})$, fine $(<30 \mu \mathrm{m})$ and non-pigmented hair, this hair synthesis by non-sexual hair follicles which does not affect by hormones (Buffoli et al., 2014; Cooper, 2015). Hair that characterizes as thicker $(>60 \mu \mathrm{m})$, longer $(>2$ $\mathrm{cm}$ ) and pigmented are called terminal hairs and can be found on the scalp, eyebrow, eyelash, armpit and pubic area (Cooper, 2015). Human hair classified using the ethnic subgroup which is African, Asian and European however recently the classification had expanded to eight main subgroups. Usually, the classification of hair involves the measurement of the three main parameters such as the curve diameter, the curl index and the number of waves (Buffoli et al., 2014; Cooper, 2015).

\section{Growth cycle of hair}

Hair growth is a continuous and cyclical process that control by the changes in the activity of hormones in individual hairs on the body at different stages of the growth cycle. The hair growth cycle can be divided into three significant difference phases, involve anagen phase, catagen phase and telogen phase (Buffoli et al., 2014; Erdoğan, 2017; Murphrey et al., 2020). All the phases would have different duration can depend on the location of the hair, individual age, hormone status and also an individual nutritional level.

Figure 1 shows the growth cycle of hair on the scalp. The anagen phase is also known as the growing phase of the hair follicle that can last for several years and about $85 \%$ to $90 \%$ of scalp hair is in this phase (Cooper, 2015; Erdoğan, 2017). This phase is characterized by the formation of the hair shaft stick out or protrude from the surface of the skin. During this phase, hair can grow approximately one centimetre every 28 days, short anagen phase would have difficulty growing hair beyond some length due to short anagen phase and this would be opposite to person that has long anagen phase (Randall and Botchkareva, 2008). Eyelashes, eyebrows, leg and arm hair are shorter than the hair on the scalp due to this hair have very short anagen phase that lasts only 30 to 45 days as compared to scalp hair that can last for several years. Fibre length or hair length often depends on the duration of the actively growing phase of the follicles (Erdoğan, 2017). In the anagen phase, there is some article that divided this phase more into six stages of 
anagen. The early phase of anagen is where hair progenitor cells start to proliferate and envelop the growing dermal papilla and begin to differentiate into the hair shaft and inner root sheath (Buffoli et al., 2014; Erdoğan, 2017). In mid anagen phase where the hair starts to show pigment due to melanocytes in the hair matrix and start to develop newly formed hair shaft. The end of the anagen phase, full restoration of hair fibreproducing unit achieved were characterized by the formation of the epithelial hair bulb surrounding the dermal papilla and the new hair shaft also start to appear from the skin surface (Randall and Botchkareva, 2008; Buffoli et al., 2014; Erdoğan, 2017). Activity that involves hair shaft synthesis and pigmentation can only take place in the anagen phase. The new hair bulb is formed at the base of the follicle and inside the hair bulb, specialized dermal papilla cells start to grow new hair shaft. If the old hair has not been shed, the newly growing hair helps push the old hair out of the follicle (Randall and Botchkareva, 2008). During anagen, there would have active signalling exchange activity occur between the epithelial cells of the hair bulb and the fibroblasts of the dermal papilla that aid in hair growth and proliferation (Randall and Botchkareva, 2008).

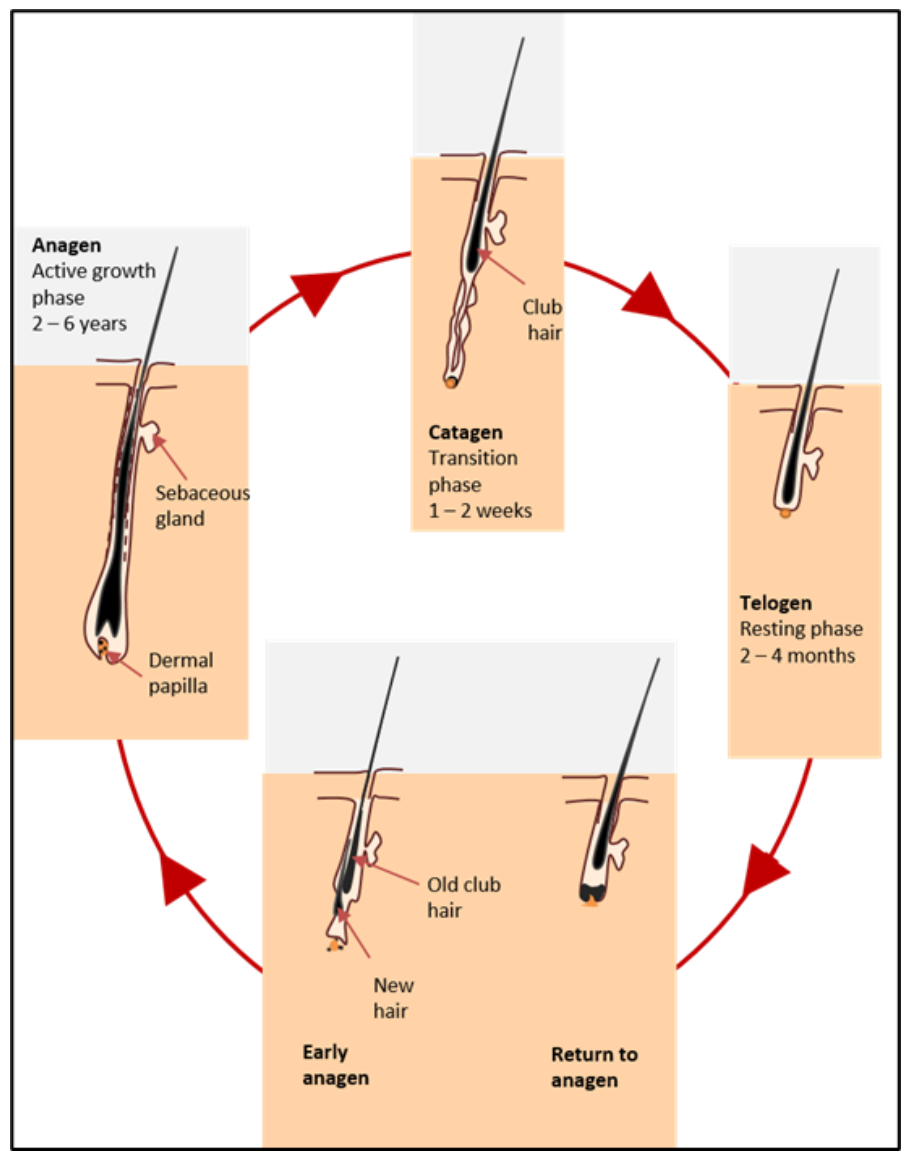

Figure 1. Normal hair growth cycle

The phase where the hair stops growing and hair follicles start to shrinking known as catagen phase. This phase is also known as regression period that lasts for two to three weeks. The lower part of hair follicle break apart and the hair follicles need less nourish from the blood supply. The first sign of the catagen phase is the termination of the melanogenesis in the hair bulb (Cooper, 2015). At the end of the anagen phase, the mitotic activity of the hair matrix cell diminished and all the differentiation and proliferation of hair matrix keratinocytes decrease significantly (Buffoli et al., 2014). Also include the pigment-producing activity of melanocytes stops and hair shaft production is completed. During the catagen phase, the follicles compartment involved in hair production is reduced to sizes that can allow the regeneration for the next hair cycle after receiving stimulation (Randall and Botchkareva, 2008). The specialized structure is known as club hair, that characterize with outer root sheath shrinks and attaches to the root of the hair.

Telogen phase takes over after the catagen phase, also known as the resting phase where the hair does not grow and the dermal papilla stays in the resting phase that lasts for months. This phase accounts for $10 \%$ to $15 \%$ of all hair and the hair follicle is at rest and club hair completely formed (Randall and Botchkareva, 2008; Buffoli et al., 2014; Cooper, 2015). The telogen phase is characterized by lack pigment-producing melanocytes, the hair shaft transformed to club hair and at the end shed off. The follicles will remain in telogen phase until the hair germ receive and responsive to the signal for initiating anagen phase from the dermal papilla (Lourith and Kanlayavattanakul, 2013). A solid, dry and has white material at the root of the hair will revel when the hair is pulled that in this telogen phase.

\subsection{Common hair problem}

Recently, many studies have proved that hair disorder may cause psychological affliction where the resultant effects may worsen the hair problem scenarios (Sengar et al., 2018). This is due to the facts that hair is an appendage which correlated with the brain in many ways. For example, when the brain in a stressful event, it may alter the adaptive immunity of the skin and hair where consequently this may lead to the premature death of the hair. Therefore, identifying the common hair disorder that affected in the human population may play a vital role in preventing these undesirable effects. Hence, the main problems that may possibly be associated with hairs are premature greying of hair (PGH), dandruff and also hair loss (balding) (Patel, Sharma, Chauhan, Thakur and Dixit, 2015).

Hair loss or alopecia is a common dermatological issue that can affect millions of human population of all ages and both gender, male and female. Frequently, alopecia has been found to be associated with a significant adverse effect or reduction of psychological and self-esteem (Montgomery et al., 2017). 
Consequently, this may lead to psychological problems such as depression and anxiety, thus it may negatively impact the quality of life as well (Phillips et al., 2017). There are several types of hair loss including androgenetic alopecia (AGA), alopecia areata (AA), alopecia totalis (AT), Alopecia Universalis (AU), cicatricial alopecia (CA), senescent alopecia (SA), traction alopecia (TA) and telogen effluvium. The summary of the etiology and histopathology for each type of hair loss were illustrated in Table 1 (Daniels et al., 2019).

\section{Androgenetic alopecia (AGA)}

Androgenetic alopecia (AGA) is the most common type of progressive hair loss that may affect both men and women. This condition is called male pattern hair loss or common baldness in men and as female pattern hair loss in women (Fabbrocini et al., 2018; Lulic et al., 2017). Basically, the histopathology of female pattern hair loss often can be seen with the diffuse thinning of the hairs in the frontal and parietal region (Ramos and Miot, 2015). Besides that, for the male-pattern baldness is hair loss occurs on the temples and crown of the head with sparing of the sides and back which is also known as "M"- shaped pattern. This pattern reflects the distribution of androgen-sensitive follicles in most people that are usually starting at puberty (Springer et al., 2003).

Alopecia in these cases is characterized by shortening of the anagen phase and continuous miniaturization of sensitive hair follicles (Springer et al., 2003). As the results, the thin terminal hair transformed to a fine vellus that may lead to the bald appearance. Additionally, studies have demonstrated that high production of androgen, dihydrotestosterone (DHT) may play a significant role in causing AGA as this may cause miniaturisation of hair follicle and its subsequent effect (Lolli et al., 2017). The study also has suggested that the androgen receptor and the metabolism of androgen are two factors that can influence the production of DHT. The 5 $\alpha$-reductase Type- 2 enzyme is an essential catalyzer in metabolism of the androgen by converting the testosterone to dihydrotestosterone (DHT) (Lulic et al., 2017).

\subsection{Causes of androgenetic alopecia (AGA)}

Hereditary thinning is also known as androgenetic alopecia (AGA), the most common types of alopecia that may affect in both men and women recently. There are ample of genetic factors may possibly play a role in causing the hereditary thinning. However, only one gene has been identified and confirmed by the scientific

Table 1. The summary of the different types of alopecia, their etiology, and manifestations as well as the typical proscribed treatment (Daniels et al., 2019).

\begin{tabular}{llll}
\hline Alopecia progenies & Etiology & Histopathology & Treatment \\
\hline
\end{tabular}

Female - diffuse hair loss (Ludwig), often Topical Minoxidil (2\%)

Male - often manifests as a receding

Androgenic

Alopecia (AGA)
Androgen dependent (testosterone/ DHT) follicle miniaturisation hairline and hair loss in the vertex region Topical Minoxidil (5\%) (Hamilton-Norwood)

Oral Finasteride/ Dutasteride

Hair transplantation

Alopecia Areata

(AA)

Alopecia Totalis

(AT)

Alopecia

Universalis (AU)

Inflammation of the upper part of

Cicatricial Alopecia the HF leading to irreversible

(CA) damage of the hair bulge and the loss of stem cells.
Regions of 'patchy' hair-loss, which predominantly manifest in the scalp.

Topical Minoxidil (5\%)

Topical or oral

corticosteroids

Complete absence of terminal scalp fibres Wig/ Hair transplantation

Complete absence of scalp and bodily fibres

Permanent loss of hair in the affected patches, visible scarring may occur

Topical or oral antibiotics,

Scalp reduction surgery

Hair transplantation

Stochastic 'ageing' of the follicle

Senescent Alopecia caused by UV, reactive oxygen

(SA)

species and other endogenous and exogenous stressors

Persistent or significant strain to the follicle, caused by particular grooming practices (Inc. braiding,

Traction Alopecia (TA)

Numerous 'triggers' including

Telogen Effluvium

(TE)

Gradual decrease in fibre-diameter and Topical Minoxidil

the number of anagen-follicles, resulting Cosmetic intervention

in a diffuse hair-loss across the entirety of the scalp (non-androgenic)

Topical antioxidants

Scarring of follicles and hair-loss at the Acute: anti-inflammatory frontotemporal margin of the scalp

Chronic: Hair transplantation
Non-scarring diffuse hair-loss, characterized by the simultaneous shedding of telogen fibres
Cosmetic interventions: topical antioxidants and $5-\alpha$ reductase inhibitors 
studies, androgen receptor (AR) gene (Ellis et al., 2002). Initially, this gene is essential in providing the instructions for producing a protein called androgen receptors. Nevertheless, variation in the AR gene is associated with an elevated risk of AGA. The changes might the results from small variation in the type or numbers of DNA building blocks that make up the AR gene (Ellis et al., 2002). Many research have believes

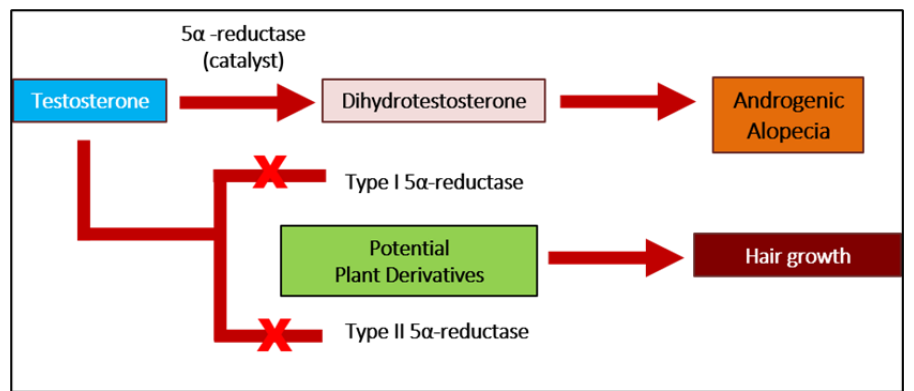

Figure 2. Schematic diagram of inhibition of $5 \alpha$-reductase from plant derivatives in androgenic alopecia management

that the variation may increase the activity of the androgen receptor in the scalp, though the actual mechanism still remains unclear. These genetic variation is happening more frequently in men with early age of hair loss. Additionally, studies suggest that having closed relatives with patterned hair loss can increase the risk factor for developing the condition.

Androgen is a hormone that can be produced naturally in the human body and usually found higher in men (Davey and Grossmann, 2016). The key pathophysiological features of male androgenic alopecia are alteration in hair cycle development, follicular miniaturization and inflammation (Cranwell and Sinclair, 2016). Testosterone and dihydrotestosterone are the major androgens that regulate hair growth. Dihydrotestosterone (DHT) has approximately a fivefold greater affinity for the androgen receptor than testosterone (Kaufman, 2002). In hair loss patients, the $5 \alpha$-reductase enzyme acts as a catalyst that converts testosterone, which is the primary androgen, to the more potent androgen, DHT which makes the hair follicle miniaturise and shed hair (Olsen et al., 2006).

Due to the effect of the potent androgen (DHT), their normal hair starts as long, thick and pigmented, then changes to be thin, short and less pigmented at the end of the process. The new hair colour becomes less pigmented from one cycle to another, the hair shaft becomes thinner, and there is the appearance of baldness. Two isozymes participate in androgen synthesis; namely, Type I $5 \alpha$-reductase isozyme which is present in the skin, hair follicles and sebaceous glands, liver, prostate, and kidney and Type II $5 \alpha$-reductase isozyme which is present in hair follicles, male genitalia and the prostate (Russell and Wilson, 1994; Kandavilli et al., 2010). Both these isozymes are involved in steroid metabolism in particular areas and interact with the androgen receptors. In androgen synthesis, the $5 \alpha$-reductase enzyme acts as a catalyst converting testosterone to the more potent dihydrotestosterone. By introducing a $5 \alpha$-reductase inhibitor, the conversion of testosterone to DHT can be decreased and help in reducing hair loss (Figure 2).

For instance, dihydrotestosterone (DHT) may interfere with the Wnt signalling pathway in the hair (Ramos and Miot, 2015). Initially, the Wnt pathway is important mechanism that use to maintain the anagen phase in the body by inducing the dermal papilla cells. Hence, the disruption of Wnt pathway by the presence of excess DHT may interfere with the hair growth cycle. The androgenic imbalance in the body can be due to several reasons. For example dysregulation of the hypothalamic-pituitary-adrenal cortex, polycystic ovary syndrome (PCOS), hormonally-active tumours and idiopathic hirsutism (Urysiak-czubatka et al., 2014).

\subsection{Treatment and management of hair loss}

Medication that currently has claims their indication to treat AGA have target a steadily growing market worldwide. However, the availability of specific therapies is still deficient and concomitant with several side effect. Frequently, the treatment status for alopecia is based on etiology and progression (Patel, Sharma, Chauhan, Thakur and Dixit, 2015). The major nonsurgical therapeutic strategies for hair growth promotion are including angiogenesis (through endogenous substances), androgen antagonism, vasodilation through potassium channel opening $5 \alpha$-reductase inhibition and modulation of the hair cycle. There are two US FDAapproved synthetic drugs finding that can be used for the treatment of androgenic alopecia including 2-5\% topical solution of minoxidil (useful in both male and female pattern baldness) and $1 \mathrm{mg}$ of finasteride (useful in male pattern baldness), however, the presence of side effects have reduced their usage (Adil and Godwin, 2017). The side effects associated with the use of these synthetic compounds include erythema, scaling, pruritus, gynecomastia, dermatitis, itching or skin rash (Etminan and Member, 2016; Patel, Sharma, Chauhan, Thakur and Dixit, 2015). Recently, another medication such as 0.5 $\mathrm{mg}$ of dutasteride has been approved and used for the treatment of AGA in a few countries including Korea and Mexico (Kelly et al., 2016). Studied have revealed that dutasteride may help to improve hair growth and reverse the androgenic alopecia. Nevertheless, the usage of the medication in therapies of alopecia may also be associated with sexual dysfunction side effect including decreased libido and ejaculation dysfunction (Tsunemi et al., 2016). Hence, this leads to an increased interest of 
researches in finding alternative treatments to overcome the hair loss problem with fewer side-effects such as formulation containing herbs or their active constituents.

Nowadays, many studies have proven that natural drugs made from plant derivative may give a promising impact to treat the AGA (Herman et al., 2017). Ample of herbal topical or natural product formulations have been marketed worldwide to prevent hair loss or promote hair growth. There are numerous advantages of using natural based compound. For examples, improve patient compliance, fewer side-effects, and easy availability, low -cost, more than one mode of biochemical action for hair loss treatment and some of it may also work synergistically with the drugs to reduce the hair loss. Based on the previous study, Karatas et al., (Karatas et al., 2016) found that treatment with topical $5 \alpha$ reductase inhibitors and supplementation of Vitamin C and omega3 fatty acids are likely appeared to be the most appropriate treatment agents for the management of hair loss. Therefore, a lot of researches are looking for new herbs and active constituents that could be potentially used as alternative tools for the drugs in therapies of alopecia. In this review, some of the plants or herbal sources that have been found could be used for the treatment of AGA will be discussed.

\subsubsection{Avicennia marina}

Avicennia marina (A. marina) is a pioneer tree species of the mangrove forest ecosystem that widely distributed along with the tropical and subtropical environment. This plant belongs to the family Acanthaceae and commonly called grey or white mangrove (Jain et al., 2016). A. marina exhibits various pharmacological activities that have been used in many countries as dermatologic disorder treatment, such as Egypt (Zhu et al., 2009; Jain et al., 2014). Extraction of the $A$. marina contains avicequinone $\mathrm{C}$ compound using methanolic heartwood extract shown potent activity of inhibition of $5 \alpha$-reductase enzyme and shown decrease in dihydrotestosterone production which can be used in the treatment of androgenic alopecia (Jain et al., 2014; Yardimci, 2017). Among all thirty Thai medicine plant, A. marina has high $5 \alpha$-reductase inhibitor and cause reduce dihydrotestosterone production about $52 \%$ as compared to other medicine plants tested (Jain et al., 2016).

\subsubsection{Boehmeria nipononivea}

Boehmeria nipononivea, B.nivea is a perennial herb that belongs to the family of Urticaceae (Ancora, 2009). The chemical constituents that have been found in the plant including 10\%-30\% fatty acid, $10 \%$ chlorogenic acid, linoleic acid, protocatechuic acid, caffeic acid and $\alpha$ -linolenic acid (Patel, Sharma, Chauhan, Thakur and Dixit, 2015). Studies have demonstrated that the plants may also have various pharmacological properties and used as medicines such as antioxidant, antidiabetic, hepatoprotective and anti-cancer and to be effective in neurodegenerative diseases (Marjahan et al., 2018). The acetone extract of $B$. nivea reported having both potent $5 \alpha$ - reductase inhibitory effect and hair re-growth promotion effect on mice (Nahata, 2017). The inhibitory effect of $5 \alpha$-reductase is due to the presence of fatty acids in the plant. For example $\alpha$-linolenic, linoleic, palmitic, elaidic, oleic, and stearic acids (Rondanelli et al., 2015).

\subsubsection{Camellia sinensis}

Camellia sinensis in the family of Theaceae is one of the common plants since ancient times, especially in China. Extraction from the Camellia sinensis leaves is known as green tea that grown in subtropical climates and is a popular drink worldwide. There are many pharmacological effects that have been shown such as ant metastatic, anticancer, anti-inflammatory, antidiabetic, antibacterial, antiviral and antioxidant effects (Patel, Sharma, Chauhan, Thakur and Dixit, 2015; Yardimci, 2017). Green tea also used in many dermatological diseases such as acne, rosacea, keloids, genital warts and atopical dermatitis (Zink and TraidlHoffmann, 2015). Besides, green tea also reported has the activity of actively inhibiting $5 \alpha$-reductase that can be used for the treatment of androgenic alopecia (Yardimci, 2017). It thought that green tea contains epigallocatechin-3-gallate (EGCG) which a major polyphenol that can give antioxidant and anti-cancer effect (Patel, Sharma, Chauhan, Thakur and Dixit, 2015). Reported that the tea polyphenolic EGCG compound can stimulate human hair growth when it tested among mice that show positive significant elongation of hair follicle (Amin et al., 2014). Apart from EGCG compound present that gives pharmacological effect, there also has catechins in the green tea that can also quickly reduce the $5 \alpha$-reductase that can act as treatment and prevention agent for hair loss problem (Zink and Traidl-Hoffmann, 2015).

\subsubsection{Citrullus colocynthis}

Citrullus colocynthis herb in family Cucurbitaceae that grow in the most barren environment is one of plant that recommended in much traditional literature that can act as a growth promoter for hair. This plant is also known as Indrayam in India that also recommended in Indian traditional medicine to treat hair loss problem (Roy et al., 2007; Yardimci, 2017). The fruit from this plant is the part of the plant that has constitutes that has promising effects to hair problem. The dried pulp of the 
unripe with full-grown fruit freed is used to treat hair loss problem. Besides that, the oil from the seeds of the plant also has reported can help to control premature falling of hair and greying of hairs (Patel, Sharma, Chauhan, Thakur and Dixit, 2015). The fruit has a pungent smell, bitter and has a purgative characteristic. Extraction of $C$. colocynthis can act as antihelmintic, antipyretic, can treat ulcers, asthma, bronchitis, enlargement of the spleen, dyspepsia, constipation, anaemia and throat disease (Roy et al., 2007; Dhanotia et al., 2011). This plant also has several pharmacological effects such as antibacterial, hypoglycemic, immunostimulating and antiandrogen beside act as hair promoting effect (Yardimci, 2017).

\subsubsection{Curcuma aeruginosa}

Curcuma aeruginosa or cucurmin (Zingiberaceae) is traditional medicines that commonly used in Thailand for gastrointestinal disturbances, uterine pain, postpartum uterine, and perimenopause bleeding (Srivilai, Waranuch, Tangsumranjit et al., 2018). Preclinical studies have suggested that $C$. aeruginosa may have several medical indications including antimicrobial, anti-inflammatory, antipyretic and analgesic properties (Srivilai, Nontakhot, Nutuan et al., 2018). Besides that, many research also have revealed that the curcumin able to inhibit $5 \alpha$-reductase, this is due to the presence of sesquiterpenes compound of which the germacrone was most potent (Herman et al., 2017). Recently, studies have proved that co-application of curcumin along with minoxidil may enhance the reversion of androgen-induced alopecia compared to the substance applied alone (Srivilai, Waranuch, Tangsumranjit et al., 2018). This is due to C. aeruginosa may help increase the epidermal penetration of minoxidil, thus improving the delivery of the drugs into the target sites.

\subsubsection{Cucurbita pepo}

Pumpkin seed oils also are known as Cucurbita pepo (C. pepo) is belong to the family of Cucurbitaceae and (Dhariwala and Ravikumar, 2019). Frequently the oils were extracted from the oval seeds of $C$. pepo and it has been found that the solvent extraction method is one of the most efficient with $98 \%$ of the oil was obtained (Cho et al., 2014). There are various chemical constituents found in the pumpkin seeds oil including fatty acids, sterols, phytoestrogen, and tocopherols. The inhibition of $5 \alpha$-reductase and anti-androgenetic effect of the pumpkin seeds oil is due to its high content of phytosterols. Studies have reported that pumpkin seed oils may show the inhibition of $5 \alpha$-reductase properties with a dose of $400 \mathrm{mg} /$ day for 24 months (Dhariwala and Ravikumar, 2019). According to the study, the presence of the phytosterols and as well lipid in the pumpkin seeds oil may synergistically treat the AGA. C. pepo has been used traditionally by many countries to treat numerous diseases. For instances anti-inflammatory, analgesic urinary, disorders antiviral, antidiabetic, anti-ulcer, and antioxidant (Perez Gutierrez, 2016).

\subsubsection{Cuscuta reflexa Roxb}

Cuscuta reflexa Roxb is a parasitic plant fit into the family of Convolvulaceae (Yardimci, 2017). The plants have a low level of chlorophyll and they cannot produce their own food by photosynthesis (Saini et al., 2018). Therefore, they completely depend on the host plant in order to obtain foods and nutrients. There are various chemical constituents obtained in the plants may have ample of pharmacological functions. For instances cuscutin, cuscutalin, sitosterol, luteolin, bergenin, kaempferol and others (Singh et al., 2016). C. reflexa has been used traditionally as numerous purposes of treatment such as antitumor, antimicrobial, hepatoprotective, anticonvulsant, antioxidant, relaxant and spasmolytic action, positive inotropic and cardiotonic activities, cholinergic action, anti-HIV, antisteroidogenic, hypoglycemic, diuretic, anti-inflammatory and induced alopecia have been previously reported (Dutta et al., 2017; Yardimci, 2017). Researches have revealed that the petroleum ether extract solution of $C$. reflexa can inhibit the 5a-reductase and as well as the conversion of testosterone to DHT, consequently, this may help in reversing the androgen-induced alopecia (Pandit et al., 2008).

\subsubsection{Glycyrrhiza glabra}

Glycyrrhiza glabra, G. glabra or licorice (family of Leguminosae) have been used in Chinese herbal medicine with long usage history and it is usually can be obtained from root and stem of the plant (Kowalska and Kalinowska-Lis, 2019). Many studies have demonstrated that licorice can be used as an alternative treatment for the AGA (Utami et al., 2017; Pastorino et al., 2018). This is due to the facts that the plant may show antagonizing testosterone effect through the presence of glycosides, terpenoid, phenolics, and flavonoids. Besides that, the existence of phytosterols in the licorice may also help to inhibit the 5a-reductase enzyme, hence reducing the production of DHT and as well as AGA. In another study also have revealed that the hydro-alcoholic extract of G. globra in 2\% concentration showed a better hair growth activity compared to $2 \%$ of standard minoxidil. Hence, this proved that the plant has a potent hair growth activity (Pandey et al., 2017). Additionally, other pharmacological activities of $G$ glabra are anti-tussive, anti-demulcent, antioxidant, anti-malarial, anti-fungal, immuno-stimulatory, anti-ulcer, anti-dyslipidemia, anti- 
thrombotic, skin lightening and anticancer properties (Pandey et al., 2017; Al-Snafi, 2018).

\subsubsection{Sophora flavescens Aiton}

Sophora flavescens Aiton root extract belongs to the family of Leguminosae and commonly used in traditional medicine (Lee et al., 2018). It has been used to treat various of disease including viral hepatitis, cancer, viral myocarditis, heat dysentery, hemifacial, jaundice, anuresis, leucorrhoea with reddish, discharge, vulval swelling, pruritus vulvae, eczema, and trichomonas vaginalis (Yardimci, 2017). The extract may have a strong inhibitory 5a-reductase effect and anti-androgen effect that can be used in treating the problematic hair issues of AGA (Roh et al., 2002). Nevertheless, the research evidence of $S$. flavescens in suggesting the extract able to promote hair growth and treat the hair loss are lacking. Additionally, studies have demonstrated that there is two active compounds of pterocarpan derivatives in the extract may help to promote the proliferation of human hair keratinocytes, L-maackiain, and medicarpin (Takahashi et al., 2016).

\subsubsection{Phyllanthus niruri}

This plant is a small plant that grows in tropical and subtropical regions in Central and South American countries, India and East Asia. This plant of genus Phullantus is also known as 'chanka piedra', 'zhuzicao', 'dukung anak' and 'chanca piedra' (Yardimci, 2017). Phyllanthus ninuri family of Euphorbiaceae widely used in traditional medicine especially in promoting hair growth. Beside can promote hair growth, this plant also commonly used for the treatment of jaundice, asthma, hepatitis, fever, malaria, antidiabetic, analgesic, wound healing and immunomodulatory effect (Patel, Sharma, Chauhan, Thakur and Kumar, 2015; Yardimci, 2017). P. niruri can increase the activity of hair growth through the mechanism of inhibition of $5 \alpha$-reductase enzyme due to the present of lignans and terpenoids that belief can contribute to the promoting of hair growth (Patel, Sharma, Chauhan, Thakur and Kumar, 2015; Yardimci, 2017; Luliana et al., 2018). It had been identified that this plant contains phenols compound that can be lignans (methylated phenols) which play a role in inhibiting the activity of 5 $\alpha$-reductase enzyme to help in reducing the conversion of testosterone into active testosterone (dihydrotestosterone) that potentially cause hair loss (Luliana et al., 2018). A study conducted had proven that $P$. niruri ethanol extract that contains methanol as the penetration enhancer showed high activity of hair growth that has been useful for the hair loss treatment.

\subsubsection{Serenoa repens}

Serenoa repens with botanical family Arecaceae is one of a native plant in West Indian that grown in large quantities on the Atlantic southeast coast of North America (Yardimci, 2017). S. repens also commonly known as saw palmetto has been used as one of natural treatment for mild to moderate androgenic alopecia and showed to be more effective than other pharmaceutical drug treatment such as finasteride (Cho et al., 2014). Saw palmetto is extracted from the berries that reported have an inhibitory effect on $5 \alpha$-reductase enzyme. Saw palmetto not only shown activity toward the $5 \alpha$ reductase enzyme but it may also cause a decrease in dihydrotestosterone uptake by the hair follicle and decrease the binding of dihydrotestosterone to androgenic receptors (Murugusundram, 2009; Yardimci, 2017). A study conducted in a group of patient with mild to moderate androgenic alopecia were treated with saw palmetto for 24 months and the result showed improvement in hair growth compared to a group of patients that treated with finasteride (Rossi et al., 2012). The side effect of the plant as alternative treatment said to be uncommon but one that can usually found when patient taking as oral form causes mild stomach discomfort. Saw palmetto can lower the prostate-specific antigen level after 6 months of treatment that can mask the early detection of prostate cancer patient (Murugusundram, 2009).

\subsubsection{Thuja orientalis}

Thuja orientalis is an evergreen coniferous tree in the family of Cupressaceae that can be found in East Asian that has been commonly used in herbal medicine. In Eastern, this plant is also known as T. occidentlis and this plant has been used to treat any type of diseases such as respiratory, skin disorder and urinary system (Yardimci, 2017). In India and China traditional medicine claims that plants of genus Thuja have hair growth-promoting effect that used as hair growth tonic however this claims lack of scientific data of their use (Thukral et al., 2017). Therefore in the present study, these plants have been used to study its promoting effect of hair growth. This plant was found potently inhibit $5 \alpha-$ reductase that said help in treating or prevent hair loss due to the androgenic effect (Kumar et al., 2012; Lourith and Kanlayavattanakul, 2013). T. orientalis leaves are used as part of the plant that contains the promoting effect of hair growth. Kaempferol and isoquercetin are compounds that were found using hot water extract. Kaempferol is a natural flavonoid that contains potent antioxidant activity and anti-inflammatory while isoquercetin also in flavonoids group that has the effect of anti-oxidant, anti-inflammatory and protective effect 
in cancer due to it can react on lipid peroxidation (Di Camillo Orfali et al., 2016; Yardimci, 2017; Deng et al., 2019; Wei and Martirosyan, 2019). In animal studies, this plant extract can induce earlier anagen phase and help in longer the mature anagen phase that can help promote hair growth and treat hair loss. T. orientalis is one of plant derive that observed that can promote more hair growth more prominently than pharmaceutical treatment such as minoxidil (Zhang et al., 2013).

\section{Nanoparticles as one of the delivery systems potentially used for androgenic alopecia treatment}

Nowadays, many modern medicines have been derived and obtained from natural resources such as plants and herbs that were used for the purpose of health promotion and treatment of diseases (Thomford et al., 2018). This is because of their unique advantages and as well as characteristics that lead to the discovery of the plant's based drugs. For instance, it may produce lower toxicity, side effects and cost, and good therapeutic potential. Nevertheless, studies have reported that most of the plants' derivatives failed to complete the clinical trial phase due to a few problems (Thomford et al., 2018). The usage of the large-sized materials in the drug delivery poses a major concern in these issues. Therefore, the study has revealed that changing of the drug delivery system using nanotechnology or nanoparticle may play a significant role in overcoming these critical issues. Nanoparticle exhibit a remarkable ability to carry and deliver the medication into the target sites with an optimum dosage range (Carter et al., 2019). As the results, this may alleviate the therapeutic efficiency of the drugs, reduced the side effects and subsequently improved patient compliance. The main interest of this method is delivery of the plant derivatives to the follicular or transdermal site in order to improve or reverse the androgenetic alopecia.

\section{Conclusion}

Overall, most of the plant derivatives or extract used in treating androgenic alopecia are targeting the inhibition of the $5 \alpha$-reductase enzyme. In the meantime, there is no permanent treatment for treating androgenic alopecia unless the patient chooses to proceed with the hair transplantation which is quite costly. Due to patient compliance, they prefer to choose the oral or topically applied ingredients even though they know that this method is impermanent in order to manage their hair loss problem. Due to that, a lot of plants have been screened by researchers to figure out that it can be used to manage the androgenic alopecia alternatively. Combining the plant extract would be work synergistically in order to increase the inhibition of $5 \alpha$-reductase enzyme. One of the methods to target and deliver the active ingredients either by orally or topically in order to prolong the efficacy of hair loss treatment is using nanoparticles delivery system. This delivery system is suggested in order to enhance the efficacy and delivery of plant derivatives by inhibiting the $5 \alpha$-reductase enzyme which is the main catalyser in the metabolism of the androgen by converting the testosterone to dihydrotestosterone. However, this system should be further investigated especially on their safety aspect due to their size and materials used. In addition, a lack of clinical trial has been found for those using plant derivatives compound in treating androgenic alopecia. More clinical trials using plant-derived compound should be undergone in order to prove the findings.

\section{Conflict of interest}

The authors declare no conflict of interest.

\section{Acknowledgement}

The authors would like to acknowledge Malaysia Higher Institution Center of Excellence (HICoE) Grant (Vote No: 4J317), Institute of Bioproduct Development, UTM and University Industry Research Laboratory (UIRL), Universiti Teknologi Malaysia for funding this research.

\section{References}

Adil, A. and Godwin, M. (2017). The effectiveness of treatments for androgenetic alopecia: A systematic review and meta-analysis. Journal of the American Academy of Dermatology, 77(1), 136-141. https:// doi.org/10.1016/j.jaad.2017.02.054

Al-Snafi, A.E. (2018). Glycyrrhiza glabra: A phytochemical and pharmacological review. IOSR Journal of Pharmacy, 8(6), 1-17.

Amin, J., Simamora, E.L.P., Anwar, E. and Djajadisastra, J. (2014). Green tea (Camellia sinensis, L.) ethanolic extract as hair tonic in nutraceutical: Physical stability, hair growth activity on rats, and safety test. International Journal of Pharmacy and Pharmaceutical Sciences, 6(5), 94 99.

Ancora, M. (2009). Products for oral use of extracts of plants to base of boehmeria nipononivea. Patent No. EP 2111866A1. Germany: European Patent Office.

Buffoli, B., Rinaldi, F., Labanca, M., Sorbellini, E., Trink, A., Guanziroli, E., Rezzani, R. and Rodella, L. F. (2014). The human hair: From anatomy to physiology. Journal of Dermatology, 53(3), 331341. https://doi.org/10.1111/ijd.12362 
Carter, P., Narasimhan, B. and Wang, Q. (2019). Biocompatible nanoparticles and vesicular systems in transdermal drug delivery for various skin diseases. International Journal of Pharmaceutics, 555, $\quad 49-62$.

j.ijpharm.2018.11.032

Cho, Y., Lee, S.Y., Jeong, D.W., Choi, E.J., Kim, Y.J., Lee, J.G., Yi, Y.H. and Cha, H.S. (2014). Effect of pumpkin seed oil on hair growth in men with androgenetic alopecia: A randomized, double-blind, placebo-controlled trial. Evidence-Based Complementary and Alternative Medicine, 2014, 549721.

Cooper, G.A.A. (2015). Anatomy and Physiology of Hair, and Principles for its Collection. In Kintz, P., Salomone, A. and Vincenti, M. (Eds.) Hair Analysis in Clinical and Forensic Toxicology, p. 1-22. USA: Academic Press. https://doi.org/10.1016/b978-0-12801700-5.00001-7

Cranwell, W. and Sinclair, R. (2016). Male androgenetic alopecia. In De Groot, L. Chrousos, G., Dungan, K., Feingold, K., Grossman, A., Hershman, J., Koch, C., Korbonits, M., McLachlan, R., New, M., Purnell, J., Rebar, R., Singer, F. and Vinik, A. (Eds.). Endotext [Internet], p. 1-32. USA: MDText.com, Inc.

Daniels, G., Akram, S., Westgate, G.E. and Tamburic, S. (2019). Can plant-derived phytochemicals provide symptom relief for hair loss? A critical review. International Journal of Cosmetic Science, 41(4), 332-345. https://doi.org/10.1111/ics.12554

Davey, R.A. and Grossmann, M. (2016). Androgen Receptor Structure, Function and Biology: From Bench to Bedside. The Clinical Biochemist Reviews, 37(1), 3-15.

Deng, S.P., Yang, Y.L., Cheng, X.X., Li, W.R. and Cai, J.Y. (2019). Synthesis, spectroscopic study and radical scavenging activity of Kaempferol derivatives: Enhanced water solubility and antioxidant activity. International Journal of Molecular Sciences, 20(4), 975. https:// doi.org/10.3390/ijms20040975

Dhanotia, R., Chauhan, N.S., Saraf, D.K. and Dixit, V.K. (2011). Effect of Citrullus colocynthis Schrad fruits on testosterone-induced alopecia. Natural Product Research, 25(15), 1432-1443. https:// doi.org/10.1080/14786410802632820

Dhariwala, M.Y. and Ravikumar, P. (2019). An overview of herbal alternatives in androgenetic alopecia. Journal of Cosmetic Dermatology, 18(4), 966-975. https://doi.org/10.1111/jocd.12930

Di Camillo Orfali, G., Duarte, A.C., Bonadio, V., Martinez, N.P., De Araújo, M.E.M.B., Priviero,
F.B.M., Carvalho, P.O. and Priolli, D.G. (2016). Review of anticancer mechanisms of isoquercitin. World Journal of Clinical Oncology, 7(2), 189-199. https://doi.org/10.5306/wjco.v7.i2.189

Dutta, A.K., Narain, B. and Dewanjee, R. (2017). A parasitic traditional medicinal plant Cuscuta Reflexa (Amarbel): An overview. World Journal of Pharmacy and Pharmaceutical Sciences, 6(6), 664 673. https://doi.org/10.20959/wjpps20176-9310

Ellis, J.A., Sinclair, R. and Harrap, S.B. (2002). Androgenetic alopecia: pathogenesis and potential for therapy. Expert Reviews in Molecular Medicine, $4(22), \quad 1-11 . \quad$ https://doi.org/10.1017/ s1462399402005112

Erdoğan, B. (2017). Anatomy and physiology of hair. In Kutlubay, Z. and Serdaroglu, S. (Eds.) Hair and Scalp Disorders, p. 13-27. InTechOpen E-Book. https://doi.org/10.5772/67269

Etminan, M. and Member, A. (2016). Persistent Sexual Dysfunction with Finasteride $1 \mathrm{mg}$ Taken for Hair Loss. Pharmacotherapy: The Journal of Human Pharmacology and Drug Therapy, 36(11), 11801184. https://doi.org/10.1002/phar.1837

Fabbrocini, G., Cantelli, M., Masarà, A., Annunziata, M.C., Marasca, C. and Cacciapuoti, S. (2018). Female pattern hair loss: A clinical, pathophysiologic, and therapeutic review. International Journal of Women's Dermatology, 4 (4), 203-211. https://doi.org/10.1016/ j.ijwd.2018.05.001

Herman, A., Herman, A.P. and Herman, A. (2017). Topically used herbal products for the treatment of hair loss : preclinical and clinical studies. Archives of Dermatological Research, 309(8), 595-610. https:// doi.org/10.1007/s00403-017-1759-7

Jain, R., Monthakantirat, O., Tengamnuay, P. and Deeknamkul, W. (2014). Avicequinone C Isolated from Avicennia marina exhibits 5 $\alpha$-reductase-Type 1 inhibitory activity using an androgenic alopecia relevant cell-based assay system. Molecules, 19, 6809-6821.

https://doi.org/10.3390/ molecules 19056809

Jain, R., Monthakantirat, O., Tengamnuay, P. and DeEknamkul, W. (2016). Identification of a new plant extract for androgenic alopecia treatment using a non -radioactive human hair dermal papilla cell-based assay. BMC Complementary and Alternative Medicine, 16(1), 1-9. https://doi.org/10.1186/s12906 -016-1004-5

Kandavilli, S., Nalamothu, V. and Pandya, V. (2010). Topical compositions comprising 5-alpha reductase inhibitors. Patent No. US 2010/0048598 A1. 
Washington, DC, USA: US Patent Trade Office.

Karatas, F., Sahin, S., Sever, A.R. and Altundag, K. (2016). Management of hair loss associated with endocrine therapy in patients with breast cancer: an overview. SpringerPlus, 5, 585. https:// doi.org/10.1186/s40064-016-2216-3

Kaufman, K.D. (2002). Androgens and alopecia. Molecular and Cellular Endocrinology, 198(1-2), 89 -95. https://doi.org/10.1016/S0303-7207(02)00372-6

Kelly, Y., Blanco, A., and Tosti, A. (2016). Androgenetic alopecia: An update of treatment options. Drugs, 76, 1349-1364. https:// doi.org/10.1007/s40265-016-0629-5

Kowalska, A. and Kalinowska-Lis, U. (2019). 18bGlycyrrhetinic acid: Its core biological properties and dermatological applications. International Journal of Cosmetic Science, 41, 325-331. https:// doi.org/10.1111/ics. 12548

Kumar, N., Rungseevijitprapa, W., Narkkhong, N.-A.,

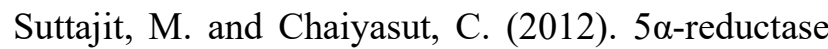
inhibition and hair growth promotion of some Thai plants traditionally used for hair treatment. Journal of Ethnopharmacology, 139(3), 765-771. https:// doi.org/10.1016/j.jep.2011.12.010

Lee, J., Jung, J., Son, S., Kim, H., Noh, Y., Min, S.R., Park, K., Kim, D., Park, S.U., Lee, H., Kim, C.Y., Kim, H., Lee, H. and Kim, H. (2018). Profiling of the major phenolic compounds and their biosynthesis genes in Sophora flavescens Aiton. The Scientific World Journal, 2018, 6218430. https:// doi.org/10.1155/2018/6218430

Lolli, F., Pallotti, F., Rossi, A., Fortuna, M.C., Caro, G., Lenzi, A., Sansone, A. and Lombardo, F. (2017). Androgenetic alopecia: a review. Endocrine, 57(1), 9 -17. https://doi.org/10.1007/s12020-017-1280-y

Lourith, N. and Kanlayavattanakul, M. (2013). Hair loss and herbs for treatment. Journal of Cosmetic Dermatology, 12(3), 210-222. https:// doi.org/10.1111/jocd.12051

Luliana, S., Desnita, R. and Rawinda, R. (2018). Formulation of hair tonic of Meniran (Phyllanthus niruri L.) ethanol extract as hair grower in male white rat (Rattus norvegicus) Wistar Strain. International Journal for Pharmaceutical Research Scholars, 7(3), 136-145. https://doi.org/10.31638/ ijprs.v7.i3.00065

Lulic, Z., Inui, S., Sim, W.Y., Kang, H., Choi, G.S., Hong, W., Hatanaka, T., Wilson, T. and Manyak, M. (2017). Understanding patient and physician perceptions of male androgenetic alopecia treatments in Asia-Pacific and Latin America. Journal of Dermatology, 44(8), 892-902. https:// doi.org/10.1111/1346-8138.13832

Marjahan, K., Hye, A., Kim, J., Ali, A., Khalil, K., Sung, W., Mi, P., Lee, K. and Hee, J. (2018). Inner morphological and chemical differentiation of Boehmeria species. Journal of Natural Medicines, 72(2), 409-423. https://doi.org/10.1007/s11418-0171164-8

Montgomery, K., White, C. and Thompson, A. (2017). A mixed methods survey of social anxiety, anxiety, depression and wig use in alopecia. BMJ Open, 7(4), 1-6. https://doi.org/10.1136/bmjopen-2016-015468

Murphrey, M. B., Agarwal, S., and Zito, P. M. (2020). Anatomy, Hair. In StatPearls [Internet]. Florida, USA: StatPearls Publishing.

Murugusundram, S. (2009). Serenoa repens: Does it have any role in the management of androgenetic alopecia? Journal of Cutaneous and Aesthetic Surgery, 2(1), 31-32. https://doi.org/10.4103/09742077.53097

Nahata, A. (2017). 5 $\alpha$-Reductase Inhibitors in the Treatment of Benign Prostatic Hyperplasia: A Review. Journal of Urology and Renal Diseases Review, 2017(7), 1-5. https://doi.org/10.29011/25757903.000053

Olsen, E., Hordinsky, M., Whiting, D., Stough, D., Hobbs, S., Ellis, M.L., Wilson, T. and Rittmaster, R.S. (2006). The importance of dual $5 \alpha$-reductase inhibition in the treatment of male pattern hair loss: Results of a randomized placebo-controlled study of dutasteride versus finasteride. Journal of the American Academy of Dermatology, 55(6), 10141023. https://doi.org/10.1016/j.jaad.2006.05.007

Pandey, S., Verma, B. and Arya, P. (2017). Review article a review on constituents, pharmacological activities and medicinal uses of Glycyrrhiza glabra. Universal Journal of Pharmaceutical Research, 2(2), 6-11.

Pandit, S., Chauhan, N.S. and Dixit, V. (2008). Effect of Cuscuta reflexa Roxb on androgen-induced alopecia. Journal of Cosmetic Dermatology, 7, 199-204.

Pastorino, G., Cornara, L., Soares, S., Rodrigues, F. and Oliveira, M.B.P.P. (2018). Liquorice (Glycyrrhiza glabra): A phytochemical and pharmacological review. Phytotherapy Research, 32, 2323-2339. https://doi.org/10.1002/ptr.6178

Patel, S., Sharma, V., Chauhan, N.S., Thakur, M. and Dixit, V.K. (2015). Hair growth: Focus on herbal therapeutic agent. Current Drug Discovery Technologies, 12, 21-42. https:// doi.org/10.2174/1570163812666150610115055

Patel, S., Sharma, V., Chauhan, N.S., Thakur, M., and Kumar, V. (2015). Evaluation of hair growth 
promoting activity of Phyllanthus niruri. Avicenna Journal of Phytomedicine, 5(6), 512-519.

Perez Gutierrez, R.M. (2016). Review of Cucurbita pepo (Pumpkin) its phytochemistry and pharmacology. Medicinal Chemistry, 6(1), 12-21. https:// doi.org/10.4172/2161-0444.1000316

Phillips, T.G., Slomiany, W.P. and Allison, R. (2017). Hair Loss: Common causes and treatment. American Family Physician, 96(6), 371-378.

Ramos, P.M. and Miot, H.A. (2015). Female Pattern Hair Loss: a clinical and pathophysiological review *. Anais Brasileiros de Dermatologia, 90(4), 529-543. https://doi.org/10.1590/abd1806-4841.20153370

Randall, V.A. and Botchkareva, N.I. (2008). The biology of hair growth. In Ahluwalia, G.S. (Ed.), Cosmetic Application of Laser and Light-Based Systems. 1st ed., p. 3-35. USA: William Andrew Inc. https:// doi.org/10.1016/B978-0-8155-1572-2.50006-3

Roh, S.-S., Kim, C.D., Lee, M.-H., Hwang, S.-L., Rang, M.-J., and Yoon, Y.-K. (2002). The hair growth promoting effect of Sophora flavescens extract and its molecular regulation. Journal of Dermatological Science, 30(1), 43-49. https://doi.org/10.1016/S0923 -1811(02)00060-9

Rondanelli, M., Perna, S., Peroni, G. and Guido, D. (2015). A bibliometric study of scientific literature in Scopus on botanicals for treatment of androgenetic alopecia. Journal of Cosmetic Dermatology, 15(2), 120-130. https://doi.org/10.1111/jocd.12198

Rossi, A., Mari, E., Scarno, M., Garelli, V., Maxia, C., Scali, E., Iorio, A. and Carlesimo, M. (2012). Comparitive effectiveness of finasteride vs Serenoa repens in male androgenetic alopecia: A two-year study. International Journal of Immunopathology and Pharmacology, 25(4), 1167-1173. https:// doi.org/10.1177/039463201202500435

Roy, R.K., Thakur, M. and Dixit, V.K. (2007). Effect of Citrullus colocynthis on hair growth in albino rats. Pharmaceutical Biology, 45(10), 739-744. https:// doi.org/10.1080/13880200701585709

Russell, D.W. and Wilson, J.D. (1994). Steroid $5 \alpha-$ Reductase: Two genes/two enzymes. Annual Review Biochemistry, 63, 25-61. https://doi.org/10.1146/ annurev.bi.63.070194.000325

Saini, P., Mithal, R. and Menghani, E. (2018). A parasitic medicinal plant Cuscuta reflexa: An overview. International Journal of Scientific and Engineering Research, 6(12), 951-959.

Sengar, S.S., Maheshwari, G., Jain, P. and Anis, S. (2018). Analysis of anxiety and depression in five hundred patients in a tertiary care hospital with common hair disorders using Hads Scale.
International Journal of Medical Science and Clinical Invention, 5(6), 3894-3898. https:// doi.org/10.18535/ijmsci/v5i6.11

Shaikh, S., Shaikh, S., Shaikh, S., Shaikh, A.A. and Saleem, S.G. (2016). Prevalence of hair loss and stress as the cause; A cross-sectional study. International Journal of Advanced Research, 4(7), 327-333. https://doi.org/10.21474/IJAR01

Singh, K., Ahmad, Z., Ahsan, F. and Shakya, P. (2016). Alopecia: Introduction and overview of herbal treatment. Journal of Chemical and Pharmaceutical Research, 8(8), 59-64.

Springer, K., Brown, M. and Stulberg, D.L. (2003). Common hair loss disorders. American Family Physician, 68(1), 93-102+107.

Srivilai, J., Nontakhot, K., Nutuan, T., Waranuch, N., Khorana, N., Wisuthiprot, W., Scholfield, C.N., Champachaisri, K. and Ingkaninan, K. (2018). Sesquiterpene-enriched extract of Curcuma aeruginosa Roxb. retards axillary hair growth: A randomised, placebo-controlled, double-blind study. Skin Pharmacology and Physiology, 31(2), 99-106. https://doi.org/10.1159/000486136

Srivilai, J., Waranuch, N., Tangsumranjit, A. and Khorana, N. (2018). Germacrone and sesquiterpeneenriched extracts from Curcuma aeruginosa Roxb. increase skin penetration of minoxidil, a hair growth promoter. Drug Delivery and Translational Research, 8, 140-149. https://doi.org/10.1007/ s13346-017-0447-7

Takahashi, T., Ishino, A., Arai, T., Hamada, C., Nakazawa, Y., Iwabuchi, T. and Tajima, M. (2016). Improvement of androgenetic alopecia with topical Sophora flavescens Aiton extract, and identification of the two active compounds in the extract that stimulate proliferation of human hair keratinocytes. Clinical and Experimental Dermatology, 41, 302307. https://doi.org/10.1111/ced.12753

Thomford, N.E., Senthebane, D.A., Rowe, A., Munro, D., Seele, P., Maroyi, A. and Dzobo, K. (2018). Natural products for drug discovery in the 21st century: Innovations for novel drug discovery. International Journal of Molecular Sciences, 19(6). https://doi.org/10.3390/ijms19061578

Thukral, S.K., Singh, S. and Sharma, S.K. (2017). Bioevaluation of hair growth potential of Thuja orientalis leaves extract. World Journal of Pharmacy and Pharmaceutical Sciences, 6(1), 1072-1088. https://doi.org/10.20959/wjpps20171-8380

Tsunemi, Y., Irisawa, R., Yoshiie, H., Brotherton, B., Ito, H., Tsuboi, R., Kawashima, M. and Manyak, M. (2016). Long-term safety and efficacy of dutasteride in the treatment of male patients with androgenetic 
alopecia. Journal of Dermatology, 43(9), 10511058. https://doi.org/10.1111/1346-8138.13310

Urysiak-czubatka, I., Kmieć, M.L. and Broniarczyk-dyła, G. (2014). Assessment of the usefulness of dihydrotestosterone in the diagnostics of patients with androgenetic alopecia. Postępy Dermatologii $i$ Alergologii, 31(4), 207-215. https://doi.org/10.5114/ pdia.2014.40925

Utami, S.M., Djajadisastra, J. and Saputri, F.C. (2017). Using hair growth activity, physical stability, and safety tests to study hair tonics containing ethanol extract of licorice (Glycyrrhiza glabra linn.). International Journal of Applied Pharmaceutics, 9 (1), 44-48. https://doi.org/10.22159/ ijap.2017.v9s1.20_25

Wei, G. and Martirosyan, D. (2019). Hair loss : A review of the role of food bioactive compounds. Bioactive Compounds in Health and Disease, 2(5), 94-125. https://doi.org/10.31989/bchd.v2i5.610

Yardimci, G. (2017). Alternative medicine for hair loss. In Kutlubay, Z. and Serdaroglu, S. (Eds.) Hair and Scalp Disorders, p. 347-373. InTechOpen E-Book.

Zhang, N., Park, D.K. and Park, H. (2013). Hair growthpromoting activity of hot water extract of Thuja orientalis. BMC Complementary and Alternative Medicine, 13, 9. https://doi.org/10.1186/1472-688213-9

Zhu, F., Chen, X., Yuan, Y., Huang, M., Sun, H. and Xiang, W. (2009). The Chemical Investigations of the Mangrove Plant Avicennia marina and its Endophytes. The Open Natural Products Journal, 2 (1), 24-32. https:// doi.org/10.2174/1874848100902010024

Zink, A. and Traidl-Hoffmann, C. (2015). Green tea in dermatology - myths and facts. JDDG - Journal of the German Society of Dermatology, 13(8), 768-776. https://doi.org/10.1111/ddg.12737 